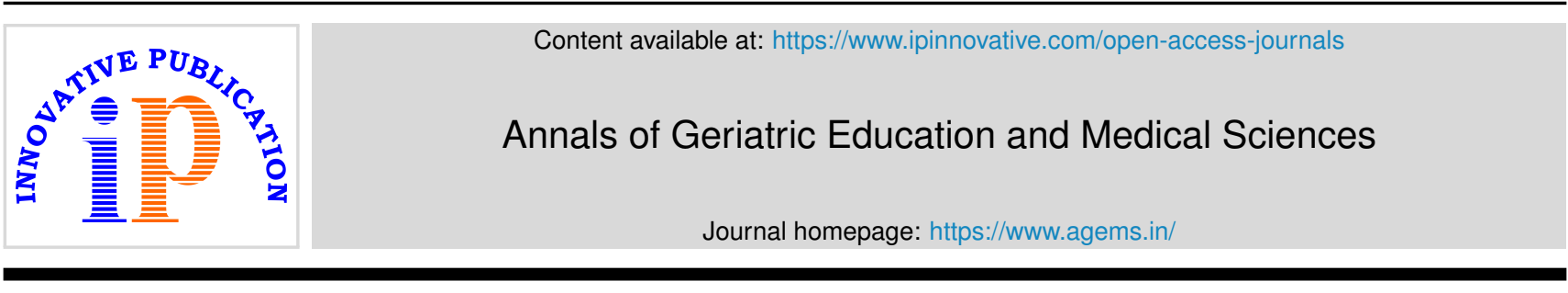

\title{
Editorial
}

\section{Constipation in elderly: Open the gut to open the mind}

\author{
Sunil Kumar ${ }^{1}$,*, Dhruv Talwar ${ }^{1}$, Charan Bagga ${ }^{1}$ \\ ${ }^{1}$ Dept. of Medicine, Jawaharlal Nehru Medical College, Datta Meghe Institute of Medical Sciences and Research, Wardha, \\ Maharashtra, India
}

\section{A R T I C L E I N F O}

Article history:

Received 26-04-2021

Accepted 20-07-2021

Available online 27-07-2021
(C) This is an open access article distributed under the terms of the Creative Commons Attribution License (https://creativecommons.org/licenses/by/4.0/) which permits unrestricted use, distribution, and reproduction in any medium, provided the original author and source are credited.
Constipation is a commonly reported bowel associated symptom in the elderly with a huge impact seen on the quality associated with life as well as expenditure on healthcare. ${ }^{1}$ Patients of the elderly age group with constipation are associated with higher psychological distress and anxiety issues. A higher prevalence of paranoid ideation, hostility and obsessive compulsive disorder is seen in the elderly who are dealing with constipation. ${ }^{2}$ Social as well a Work life are deeply affected negatively by constipation leading to a significant effect on quality of life.

Constipation can often be associated with wide range of symptoms that negatively influence the daily life of a elderly. Many patients reported to having to make effort during defecation and eliminating hard stools and feeling of incomplete evacuation or difficult stool passage and these symptoms had a huge impact on their life leading to great mental distress. ${ }^{3}$ Although, it is not a physiologic condition associated with ageing, decreased mobility in old age, rectal sensory motor dysfunction, various co morbid conditions and drug intake might be contributing to development of constipation in the elderly. From a pathologic point of view constipation may itself present as a disease or may present clubbed as a complex clinical picture in secondary forms. Primary form may be a slow transit constipation or outlet obstruction or constipation associated with IBS.

\footnotetext{
* Corresponding author.

E-mail address: sunilkumarmed@gmail.com (S. Kumar).
}

Slow transit constipation refers to a increased time taken by stool to pass through the colon and is often associated with reduced rectal sensitivity.

High and low amplitude propagated contractions promote rapid movements of intraluminal content and their presence is often associated with evacuation. Chronic constipation patients show a reduction in High amplitude Propagated contractions to less than 5 per day. Also, Gastro colic reflex is found to be deficient in the elderly contributing to the constipation. The lack of coordination between contraction of abdominal muscles and relaxation of pelvic floor muscles on straining may lead to constipation by difficult or unsatisfactory expulsion of feaces from the rectum. ${ }^{4}$ Elderly patient also report to lead a sedentary lifestyle, reduced water intake causing dehydration as well as less fiber intake in food, all leading to constipation.

Accumulation of different causes for development of constipation often leads to requirement of a multifactorial treatment approach to treat this constipation. ${ }^{5}$ Many patients often respond to conservative approach with bowel traning measures and lifestyle modification. However patients not responding to conservative line of management need to be approached with a tailored approach addressing various co morbid conditions of the elderly.

Treatment begins with non-pharmacological management which consists of lifestyle changes like having a regular daily routine with starting of the day with light physical activity. The optimal times to have a bowel 
movement are soon after walking and after meal when colon increases its motor activity. Thus, patients should be encouraged to attempt defecation first thing in the morning as well as in the post prandial intervals to make the most profit out of gastro colic reflex.

A Gradually increasing fluid intake of fluids and fibers upto $30 \mathrm{~g} /$ day is recommended. The patient must be advised to achieve this with the help of increase in fruits and vegetable intake in daily diet. Probiotics have also been shown to have efficacy with respect to relief of constipation and can be prescribed to patients on lifestyle modifications. Patients should also be educated about the adequate effort for defecation, training for straining to improve the pushing effect from the abdomen as well as practice simulated defecation by using inflated rectal balloon. Stool Softeners though widely prescribed have limited role in the treatment of constipation in the elderly. Enemas and Suppositories may be used in a effort to decrease chaces of fecal impaction however the side effects such as dyselectrolytemia and rectal mucosal damage should be kept in mind while using them. When indicated, tap water enema is the best way to go. Osmotic laxatives are agents that are hyperosmolar in nature and lead to secretion of water into the lumen of the intestine by their osmotic activity. Lactulose, lacitol and macrogol are commonly used in the elderly patients.

Lactulose is a non digestible and synthetic carbohydrate (disaccharides) that get fermented due to the bacteria found in the colonic flora causing increase in water content of the stool and leads to softening of stool thus relieving constipation. Lactulose also causes proliferation of lactobacilli by its prebiotic action leading to shortening of transit time of stool by acidification. Despite availability of a broad spectrum of laxatives it is often seen that most patients do not achieve satisfactory outcomes with the drugs that are commonly being used.

New drugs nowadays are aiming towards physiological mechanism of action are being developed to treat constipation. Among the new therapeutic agents pro secretory products (lubiprostone and linaclotide) and serotonergic agents are showing promising results.

To conclude, Constipation is a common and self reported symptom of the digestive system that is widely reported in the old age group. It is a important aspect affecting the quality of life and causing great psychological stress to the patient. A thorough assessment with careful drafting of treatment plan is highly essential in management of patient of old age group presenting with constipation. Life style modification and increasing mobility are mainstay along with help of minimum required drugs. Battling constipation in these patients yields a great benefit of a huge positive impact on their day to day life with phenomenal improvement in quality of life.

\section{Source of Funding}

None.

\section{Conflict of Interest}

None.

\section{References}

1. Higgins P, Johanson JF. Epidemiology of Constipation in North America: A Systematic Review. AM J Gastroenterol. 2004;99(4):7509. goi:10.1111/1.1572-0241.2004.04114.x,

2. Bharucha AE, Pemberton JH, Locke GR. American Gastroenterological Association Technical Review on Constipation. Gastroenterol. 2013;144(1):218-38. do1:10.1053/].gastro.2012.10.028

3. Kumar S, Sharma DK, Auluck S. Band Gap Tuning Of ZnO 1-3x N 2x F x Alloys: A First Principles Study. Mater Today Proc. 2017;4(4):57005. doi:10.1016/1.matpr.2017.06.033

4. Wald A, Scarapignato C, Kamm MA, Lissner SM, Hwlfrich I, Schuijt $\mathrm{C}$, et al. The burden of constipation on quality of life: results of a multinational survey. Alimentary Pharm Ther. 2007;26(2):227-36. do1:10.1111/.1365-2036.2007.033/6.X.

5. Gliav A, Lindberg G. Quality of Life in Patients with Different Types of Functional Constipation. Scand J Gastroenterol. 1997;32(11):1083-9. [0.: $0.3709 / 00365529709002985$

\section{Author biography}

Sunil Kumar, Professor

Dhruv Talwar, Post Graduate Resident

Charan Bagga, Post Graduate Resident

Cite this article: Kumar S, Talwar D, Bagga C. Constipation in elderly: Open the gut to open the mind. Ann Geriatrics Educ Med Sci $2021 ; 8(1): 1-2$. 